\title{
Depth Sensitivity Analysis of high-density imaging arrays for mapping brain function with Diffuse Optical Tomography
}

\author{
Hamid Dehghani ${ }^{1}$, Brian R. White ${ }^{2}$, Benjamin W. Zeff ${ }^{2}$ and Joseph P. Culver ${ }^{2}$ \\ ${ }^{1}$ School of Physics, University of Exeter, UK \\ ${ }^{2}$ Department of Radiology, Washington University School of Medicine, St. Louis, MO 63110 \\ email:h.dehghani@exeter.ac.uk, Tel:+44 1392 264177,Fax:+44 1392261111
}

\begin{abstract}
Developing diffuse optical tomography methods for neuroimaging of humans is challenging due geometry and light level constraints. Analysis of multi-distant high-density imaging arrays shows the feasibility of imaging up to $20 \mathrm{~mm}$ depth within the adult brain.

(C)2008 Optical Society of America

OCIS codes: (170.6960) Tomography; (170.2655) Functional monitoring and imaging; (330.4270) Vision system neurophysiology
\end{abstract}

\section{Introduction}

Functional mapping of the human brain is an important aspect of cognitive neuroscience, used to study brain organization and development. Increasingly, functional neuroimaging is also being used as a diagnostic and prognostic tool in the clinical setting. Additionally, many situations are not amenable to scanner geometries, such as subjects who are in the intensive care unit, who are performing complex tasks, or who might otherwise require sedation. Diffuse optical imaging (DOI) is a methodology uniquely suited to such tasks, as it is a mobile system utilizing a small, flexible imaging cap. DOI images hemodynamic contrasts like functional magnetic resonance imaging blood-oxygen-level dependent (fMRI-BOLD) imaging; however, DOI can measure changes in oxygenated hemoglobin $\left(\triangle \mathrm{HbO}_{2}\right)$, deoxygenated hemoglobin $(\triangle \mathrm{HbR})$, and total hemoglobin $(\triangle \mathrm{HbT})$ while BOLD detects only $\Delta \mathrm{HbR}$. Despite unique strengths, however, optical imaging as a standard tool for functional mapping has been limited by low spatial resolution, a lack of volumetric localization, and instrument complexity. We have developed an optical imaging system with superior contrast-to-noise characteristics that allows higher resolution imaging than previously obtained and overcomes many of these limitations, while maintaining simple instrumentation [1].

The vast majority of DOI studies have been performed in topographic mode, in which the image is synthesized from measurements at a single source-detector pair separation and without overlapping measurements. Topographic DOI has been used extensively, for example, to image functional responses in the human visual [2] sensorimotor [3], and auditory cortices [4]. Topographic DOI has limited lateral resolution $(>2 \mathrm{~cm})$ and no depthsectioning capability, precluding spatial separation of superficial and brain signals. Because of these limitations, topography studies of the visual cortex have been limited to distinguishing contra-lateral activations, an imaging task requiring no greater than $4 \mathrm{~cm}$ resolution.
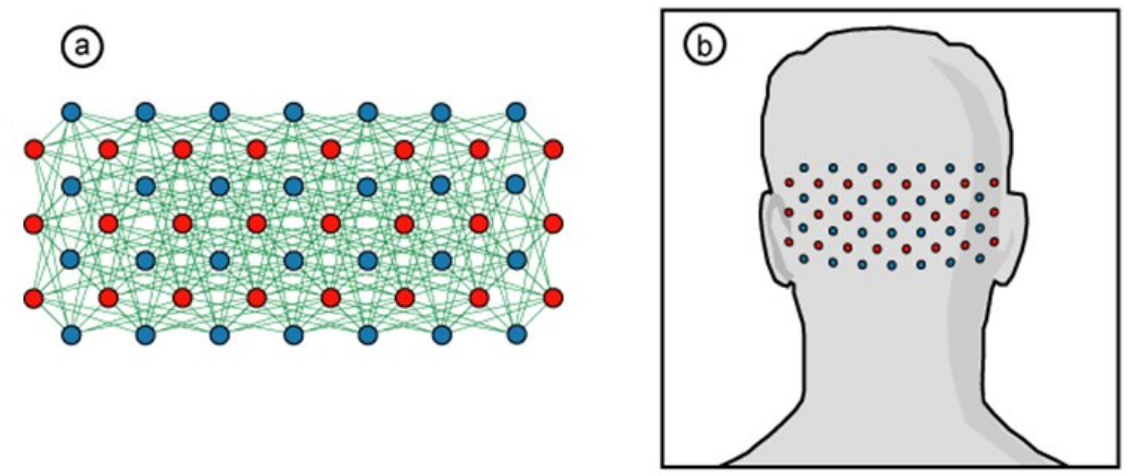

Figure 1 (a) Schematic of the high-density imaging grid, with 24 sources (red) and 28 detectors (blue). Measurement pairs are represented by green lines. (b) Schematic showing the placement of the imaging grid over the visual cortex.

In a recent study we have presented a new DOT system that has high contrast-to-noise, allowing visualization of individual activations and highly repeatable mapping within and across subjects [1]. The new 


\section{BMD27.pdf}

system has shown that with the improved spatial resolution and localization, functional responses of $1.5 \mathrm{~cm}$ in extent and shifts of less than $1 \mathrm{~cm}$ are able to be imaged. Cortical maps of angle and eccentricity in the visual field were shown to be consistent with retinotopic studies using functional magnetic resonance imaging (fMRI) and positron emission tomography (PET). These results, encouragingly demonstrated that high-density DOT is a practical, powerful tool for functional mapping of the human cortex. The system consists of high-density DOT imager using a continuous wave $(\mathrm{CW})$ instrument having 24 source positions with two near-infrared (NIR) wavelengths $(750 \mathrm{~nm}$ and $850 \mathrm{~nm}$ ) at each position. A total of 28 detector channels are used, each with a dedicated avalanche photodiode (APD) and dedicated 24-bit ADC line to eliminate switching and decreasing crosstalk. The 24 sources and 28 detectors are interleaved in a high-density array (Figure 1a), with a first-nearest-neighbor spacing of $13 \mathrm{~mm}$ and overall dimensions of $13.2 \mathrm{~cm} \times 6.6 \mathrm{~cm}$. The optical fibers are coupled to the head using a flexible, plastic cap molded to fit the back of the head over the visual cortex (Figure 1b) and held on with hook-and-loop strapping. Each detector is able to sample light from all sources, for a total of 672 possible measurements.

With the high sensitivity of the instrument, first- $(13 \mathrm{~mm})$, second- $(30 \mathrm{~mm})$, third- $(40 \mathrm{~mm})$, and fourthnearest-neighbor $(48 \mathrm{~mm}$ ) optode pairs (and greater, in certain situations) can be sampled simultaneously with light levels well above the noise floor, for a total of 348 measurements. Functional maps obtained from adult humans clearly show localized activations of visual cortex [1]. These experimental results demonstrate the feasibility of imaging the cortical tissue. In this work, we present numerical simulations using a finite element model of the adult head to study the sensitivity of the measured signal, as a function of the imaging array geometry.

\section{Methods}

The numerical model used is a 3 dimensional finite element representation of the adult head as shown in Figure 2. The mesh contains 88492 nodes corresponding to 502526 linear tetrahedral elements. Three different regions were considered, Muscle/skin, Bone and Brain whose physiological parameters are shown in Table 1. Simulations were done to calculate the total sensitivity when considering the first, second, third, and fourth-nearestneighbor optode pairs at a single wavelength of $750 \mathrm{~nm}$. The total sensitivity for each optode configuration was calculated using NIRFAST [5] assuming only $\mathrm{CW}$ data at $750 \mathrm{~nm}$ and small changes in tissue optical absorption parameters.
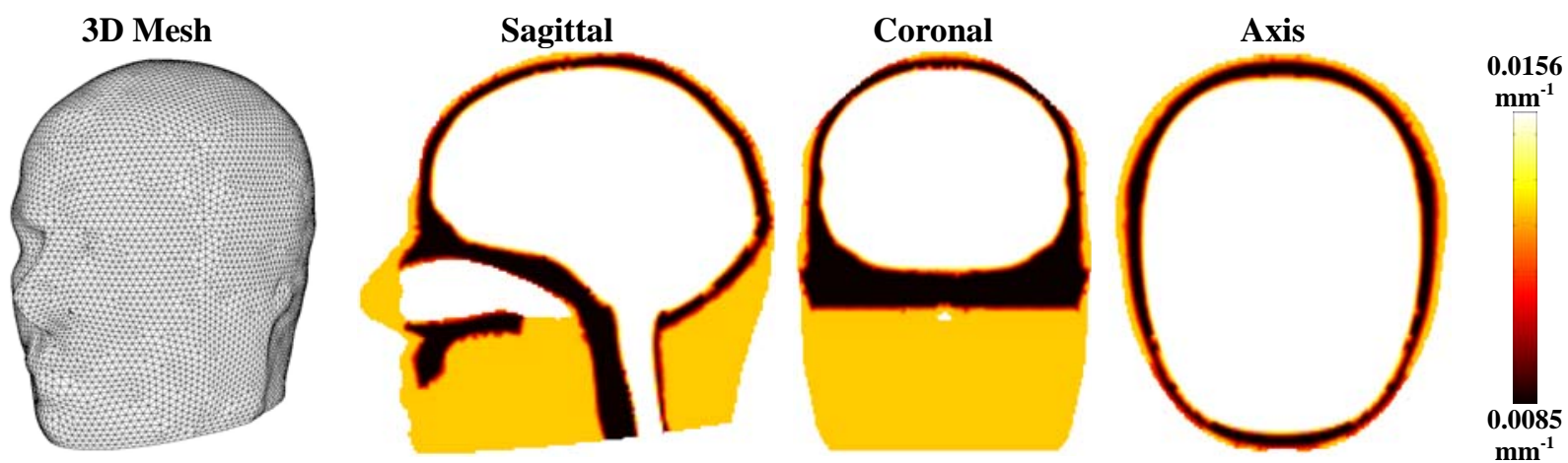

Figure 2. Three dimensional (3D) model of the adult brain and the corresponding cross-sectional maps of absorption at $750 \mathrm{~nm}$.

\begin{tabular}{|c|c|c|c|c|c|}
\hline & HbT $(\mu \mathrm{M})$ & $\mathrm{SO}_{2}(\%)$ & $\mathbf{H}_{2} \mathrm{O}(\%)$ & Scatter Size & Scatter Amplitude \\
\hline Muscle & 70 & $\mathbf{8 0}$ & 50 & 0.14 & 2.82 \\
\hline Bone & 49 & 80 & 15 & 1.4 & 1.47 \\
\hline Brain & 76 & 71 & 78 & 0.54 & 0.54 \\
\hline
\end{tabular}

Table 1. Physiological parameters used for each region of the 3D model [6].

\section{Results}

The total sensitivity calculated for each optode pair combination is shown in Figure 3 . The total sensitivity shown as percentile contours over the back portion of the axial view (Figure 2) corresponds to the mid-level of the imaging grid (Figure 1a). It is clearly evident that the sensitivity is largely dominated by the muscle and bone and using the $1^{\text {st }}$ nearest optode pair does not provide much sensitivity deep within the brain. As the number of nearest optode measurements is increased, the total sensitivity also increases as a function of depth. Specifically, given the $4^{\text {th }}$ nearest optode measurements, the total sensitivity within the brain is increased in the brain of up to more than 20 $\mathrm{mm}$, which would indicate that changes in hemodynamic contrasts at these depths can be sampled. 


\section{BMD27.pdf}
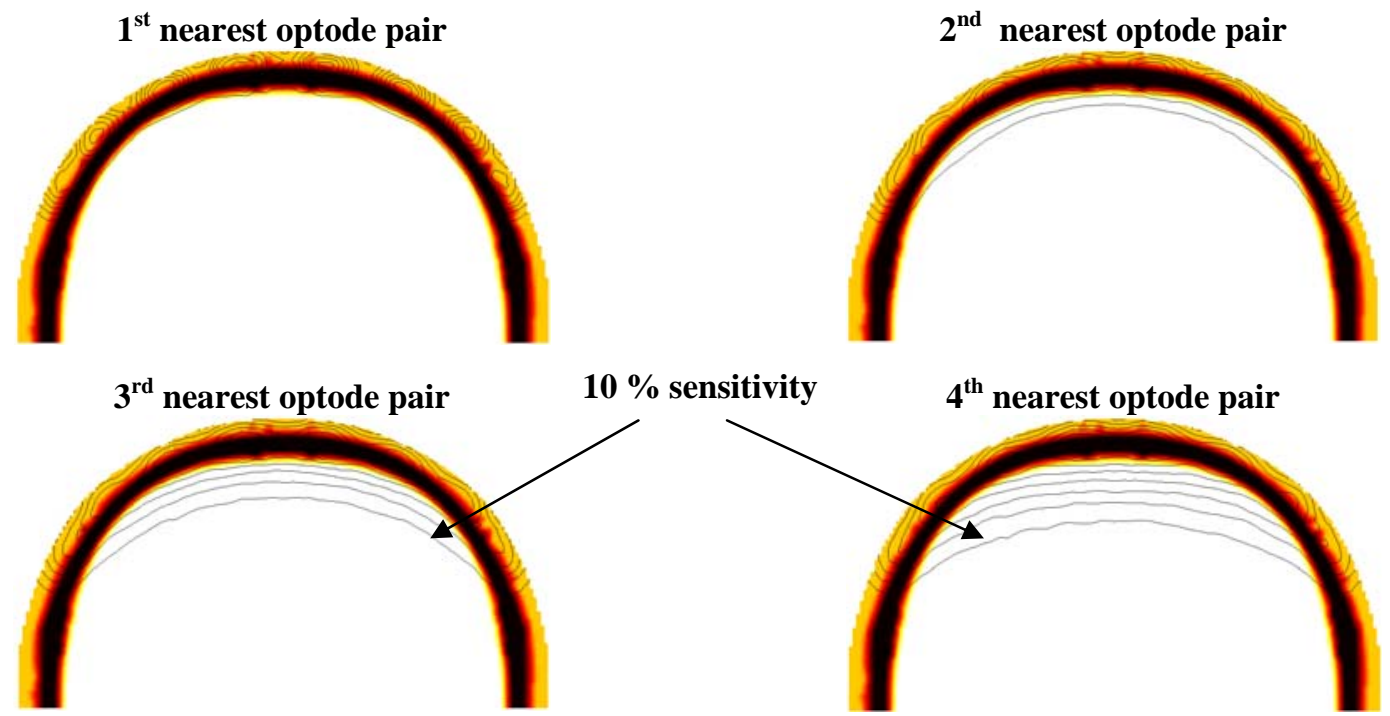

Figure 3. The total sensitivity shown as contour lines on the back portion of the axial view of the 3D adult head model. The shades in each image represent the optical absorption properties, as shown in Figure 2, and each contour line represent $10 \%$ percentile of the total sensitivity.

\section{Discussion}

In this work we have presented the sensitivity of $\mathrm{CW}$ data, as measured using our functional neuroimaging diffuse optical tomography. Specifically, using total sensitivity plot analysis on a physiologically layered 3 dimensional model of the adult head, we have calculated the total amount of sensitivity in optical absorption, at a single wavelength of $750 \mathrm{~nm}$, for a number of source and detector combination. It is seen that using only the nearest pair measurements, the sensitivity is limited to superficial layers, and specifically the bone. It is evident that for the $2^{\text {nd }}$ and $3^{\text {rd }}$ nearest optodes, the sensitivity is concentrated near the surface of the cortex. However, increasing the number of measurements to the forth nearest neighbors, it is shown that the sensitivity of measured data can be extended to as much as $20 \mathrm{~mm}$ deep within the cortex, albeit at a reduced magnitude which maybe overcome in image reconstruction using appropriate constraints, such a spatially variant regularization.

\section{Acknowledgements}

This work was supported in part by National Institutes of Health Grants K25NS4339 and R21EB7924 as well as Engineering and Physical Sciences Research Council, UK. HD would like to thank Andrew Tizzard for the finite element mesh of the adult head.

\section{References}

1. Zeff, B.W., White, B. R., Dehghani, H., Schlaggar, B. L., and Culver, J. P., Retinotopic mapping of adult human visual cortex with high-density diffuse optical tomography. PNAS, 2007. 104: p. 12169-12174.

2. Gratton, G., Corballis, P. M., Cho, E., Fabiani, M. and Hood, D. C., Shades of gray matter: Noninvasive optical images of human brain reponses during visual stimulation. Psychophysiology, 1995. 32(5): p. 505-509.

3. Franceschini, M.A., Fantini, S., Thompson, J.J., Culver, J.P., Boas, D.A.,, Hemodynamic evoked response of the sensorimotor cortex measured non-invasively with near-infrared optical imaging. Psychophysiology, 2003. 40: p. 548-560.

4. Zaramella, P., Freato, F., Amigoni, A., Salvadori, S., Marangoni, P., Suppjei, A., Schiavo, B., Chiandetti, L., Brain auditory activation measured by near-infrared spectroscopy (NIRS) in neonates. Pediatr. Res, 2001. 49: p. 213-219.

5. Dehghani, H., Pogue, B. W., Poplack, S. P., Paulsen, K. D., Multiwavelength Three-Dimensional Near-Infrared Tomography of the Breast: Initial Simulation, Phantom, and Clinical Results. Applied Optics, 2003. 42(1): p. 135-145.

6. Torricelli, A., Pifferi, A., Taroni, A., Giambattistelli, E. and Cubeddu, R., In vivo optical characterization of human tissues from 610 to $1010 \mathrm{~nm}$ by time-resolved reflectance spectroscopy. Physics in Medicine and Biology, 2001. 46: p. 2227-2237. 